\title{
Luminescence in Highly Excited InGaN/GaN Multiple Quantum Wells Grown on GaN and Sapphire Substrates
}

\author{
S. Miasojedovas ${ }^{a, *}$, S. JuRŠĖnAS ${ }^{a}$, G. KuRILČIK $^{a}$, \\ A. Žukauskas ${ }^{a}$, V.Yu. IVAnOV ${ }^{b}$, M. Godlewski ${ }^{b, c}$, \\ M. Leszczyński ${ }^{d}$, P. PERLiN ${ }^{d}$ AND T. Suski ${ }^{d}$
}

${ }^{a}$ Institute of Materials Science and Applied Research, Vilnius University Saulètekio al. 9, building III, 10222 Vilnius, Lithuania

${ }^{b}$ Institute of Physics, Polish Academy of Sciences al. Lotnikow 32/46, 02-668 Warsaw, Poland

${ }^{c}$ Dept. Math. and Natural Sci. College of Science

Cardinal S. Wyszyński Univ., Warsaw, Poland

${ }^{d}$ High Pressure Research Center, Polish Academy of Sciences Warsaw, Poland

We report on high-excitation luminescence spectroscopy in $\operatorname{In}_{x} \mathrm{Ga}_{1-x} \mathrm{~N} / \mathrm{GaN}$ multiple quantum wells grown by MOCVD over sapphire and bulk GaN substrates. High excitation conditions enabled us to achieve a screening of the built-in field by free carriers. This allowed for the evaluation of the influence of band potential fluctuations due to the variation in In-content on efficiency of spontaneous and stimulated emission. InGaN/GaN multiple quantum wells grown on bulk GaN substrate exhibit a significantly lower stimulated emission threshold and thus enhanced lateral emission. Transient and dynamic properties of luminescence indicate a significant reduction in compositional disorder in homoepitaxially grown structures.

PACS numbers: 78.45.+h, 78.47.+p, 78.67.De

*corresponding author; e-mail: saulius.miasojedovas@ff.vu.lt 


\section{Introduction}

InGaN is the key material for production of light emitting diodes that operate from the visible to UV spectral region $[1,2]$. Despite the commercialization of these devices, the luminescence efficiency suffers from a high density of dislocations due to the lattice mismatch between the substrate and active layers. The dislocations are also involved in the formation of compositional inhomogeneity [3]. Various substrates were used to avoid excessive heating of structure during the operation and penetration of dislocations into the active layer [4-6]. A significant reduction in dislocation density can be obtained in multiple quantum well (MQW) structures grown on GaN substrates [7].

Here we present results on high-excitation luminescence spectroscopy in InGaN/GaN 20-period MQWs grown over sapphire and bulk GaN substrates. High-power excitation conditions were close to those of semiconductor laser operation regime. The created carrier density was of the order of $10^{19} \mathrm{~cm}^{-3}$, which enabled us to characterize the structures under conditions of screened built-in electric field [8-10] and to analyse recombination dynamics within the tail of the density of the states.

\section{Experimental}

Samples were grown on sapphire and bulk GaN substrates. Each structure consists of 20 InGaN quantum wells separated by GaN barrier layers. GaN substrates were prepared by high-pressure technique and the MQWs were grown by metal organic chemical vapor deposition. Both samples contain a GaN buffer layer of $0.55 \mu \mathrm{m}$ thickness doped with silicon (approximately $10^{18} \mathrm{~cm}^{-3}$ ). MQWs consisting of $3.5 \mathrm{~nm}$ InGaN quantum wells (approximately $7 \%$ of indium) and $8 \mathrm{~nm}$ GaN barriers were deposited on the buffer layer. The structures were covered by a $18 \mathrm{~nm}$ GaN capping layer. The sample grown on sapphire substrate has the same structure except that a nucleation layer of AlN was grown on the substrate.

The samples were excited by the third harmonic (photon energy $h \nu=$ $3.49 \mathrm{eV}$ ) of the actively-passively mode-locked YAG: $\mathrm{Nd}^{3+}$ (yttrium aluminum garnet) laser (pulse duration of $\tau=20 \mathrm{ps}$, repetition rate $-2.7 \mathrm{~Hz}$, maximum pump energy of the third harmonic $-25 \mu \mathrm{J})$. The size of excitation spot was approximately $1.3 \mathrm{~mm}$. Luminescence was collected in backward and lateral geometries and dispersed by a $0.4 \mathrm{~m}$ grating monochromator. Toluene optical Kerr shutter was used for temporal resolution (20 ps) of the luminescence. The experiments were carried out at room temperature.

\section{Results and discussion}

Figure 1 depicts time-integrated luminescence spectra of the InGaN MQWs, measured at $1.5 \mathrm{~mJ} / \mathrm{cm}^{2}$ excitation energy density and recorded in backward 


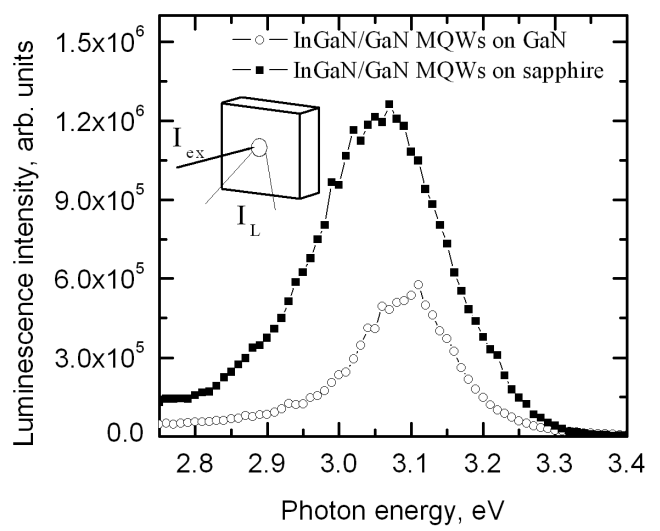

Fig. 1. Time-integrated luminescence spectra of InGaN/GaN MQWs grown on GaN (circles) and on sapphire (squares) substrates, measured at $1.5 \mathrm{~mJ} / \mathrm{cm}^{2}$ excitation energy density and recorded in backward geometry.

geometry. Both spectra show one broad luminescence band originated from the QWs region, peaked at $3.07 \mathrm{eV}$ and at $3.1 \mathrm{eV}$ for MQWs over sapphire and GaN substrates, respectively. The only difference between the two structures is the density of threading dislocations. The structure grown over bulk GaN is expected to be of a higher quality and to possess a lower density of nonradiative traps. However, the MQW grown over sapphire substrate shows more than twice intense spontaneous emission as that grown over bulk GaN. In the structure grown over sapphire, the broadening and red-shift of the luminescence band can be attributed to smearing of the band edge which results in a more extended band tail.

Figure 2 shows time-resolved luminescence spectra of the two structures observed in backward geometry for $1.5 \mathrm{~mJ} / \mathrm{cm}^{2}$ excitation energy density. The
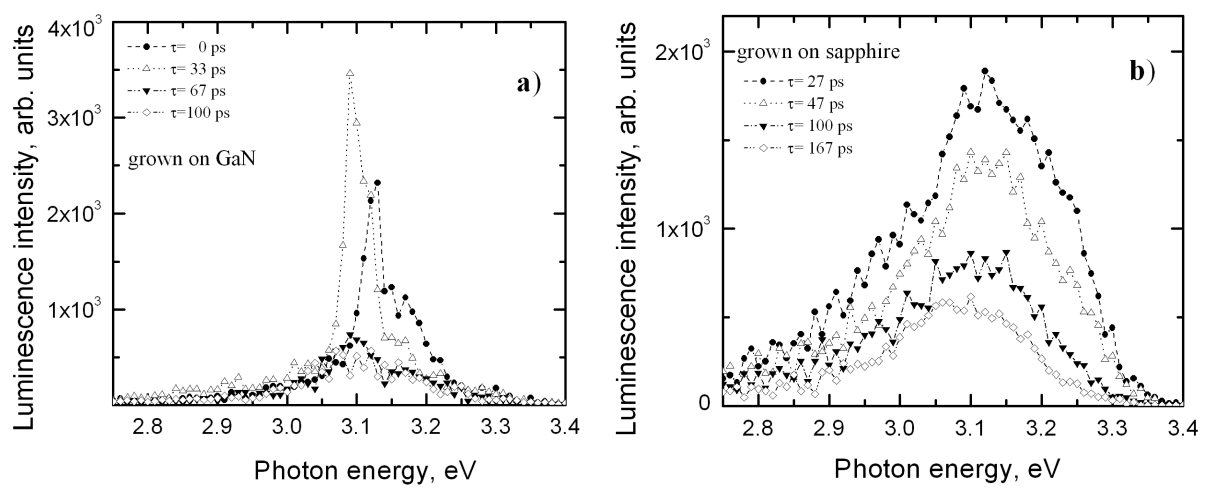

Fig. 2. Time-resolved luminescence spectra measured at $1.5 \mathrm{~mJ} / \mathrm{cm}^{2}$ excitation energy density and recorded in backward geometry for structures (a) grown on bulk GaN and (b) grown on sapphire. Delay-time (in ps) is indicated on the left-hand side. 
structure grown over GaN shows a strong narrow emission band, which is typical of amplified luminescence. Meanwhile, the structure grown over sapphire shows a broad band of spontaneous emission. A higher threshold of stimulated emission in the structure grown over sapphire can be attributed to a larger compositional inhomogeneity [3] and thus to a lower density of states [9, 10].

A transient red-shift (of about $50 \mathrm{meV}$ ) in homoepitaxial sample is observed on the initial stage (first $100 \mathrm{ps}$ ) of the relaxation. On this stage, an enhanced carrier recombination rate that is slowing down with time was observed. Such behavior indicates a screened built-in field. The initial relaxation can be explained in terms of carrier localization within random energy potential. After the excitation pulse terminates, the carriers from higher energy states can be more efficiently captured by nonradiative traps or recombine radiatively via stimulated transitions. The strong transient red-shift and the decrease in intensity are typical of luminescence in highly excited disordered systems [9]. After 100 ps, only spontaneous emission is observed for both samples [10].

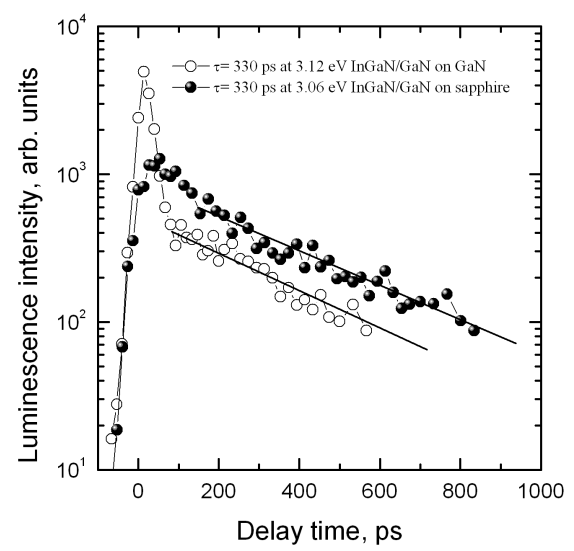

Fig. 3. Luminescence transients obtained of InGaN/GaN MQWs grown on GaN (open circles) and on sapphire (solid circles) substrates, at the peak of spontaneous luminescence band at $1.5 \mathrm{~mJ} / \mathrm{cm}^{2}$.

Figure 3 shows the luminescence transients measured at the peak positions of the corresponding time-integrated luminescence bands. Stimulated emission on the initial stage of the transients rapidly diminishes the initially created carrier density in MQWs grown over GaN. After exhaustion of stimulated emission, the remaining carrier density is lower than that in MQWs on sapphire. This accounts for a low efficiency of spontaneous luminescence of MQWs grown over GaN. On the late relaxation stage, both structures show almost the same 330 ps decay-time, which is due to nonradiative recombination of delocalized carriers.

Time-integrated luminescence dependence on excitation intensity was measured in InGaN/GaN MQWs using lateral geometry (not shown). At low exci- 
tation intensities $I_{\mathrm{e}}<0.3 \mathrm{~mJ} / \mathrm{cm}^{2}$, both samples showed one broad spontaneous luminescence band situated at $3.05 \mathrm{eV}$ and $2.97 \mathrm{eV}$ for the structure grown on GaN and on sapphire, respectively. With an increase in excitation density, a stimulated-emission peak appeared on the high-energy side of the spectrum. This peak was positioned at $3.1 \mathrm{eV}$ and $3.26 \mathrm{eV}$ for the sample grown on GaN and sapphire, respectively. This difference in energy indicates on enhanced fluctuations of the band potential in the heteroepitaxial sample. Meanwhile, the threshold for stimulated emission was lower for the structure grown over GaN (about $\sim 0.6 \mathrm{~mJ} / \mathrm{cm}^{2}$ ). The MQW structure grown on GaN also showed the lateral-luminescence efficiency that was by two orders of magnitude higher in comparison with the sample grown on sapphire substrate. This result also explains the reduction of the luminescence intensity in backscattering measurements.

Figure 4 depicts time-resolved lateral-luminescence spectra of InGaN/GaN MQW structures (the spectra are arbitrary shifted along the vertical axis). On the initial stage $(-20 \mathrm{ps}-0 \mathrm{ps})$, the sample grown on bulk GaN substrate shows one narrow band of stimulated emission, which is situated at $3.12 \mathrm{eV}$. Owing to lower joint density of states, stimulated emission starts from 0 ps delay time in the heteroepitaxial sample. The band of stimulated emission decays rapidly with the time constant below our temporal resolution $(\tau<20 \mathrm{ps})$. After $70 \mathrm{ps}$, only the spontaneous luminescence peak is observed at $3.07 \mathrm{eV}$ (the peak is red-shifted due to the reabsorption) (Fig. 4a). Meanwhile, the structure grown on sapphire substrate (Fig. 4b) shows a remarkably blue-shifted (at about $3.25 \mathrm{eV}$ ) stimulated-emission band of lower intensity.
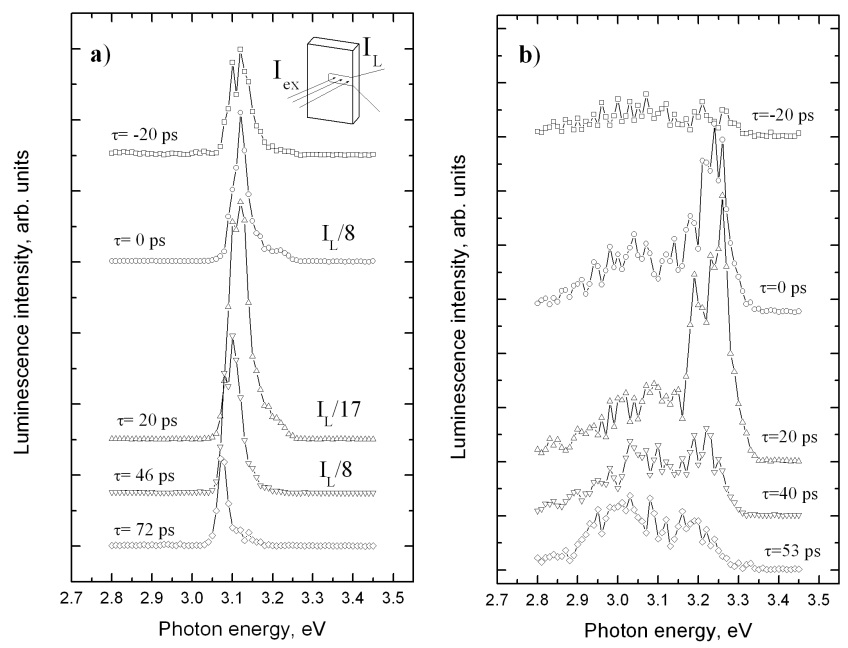

Fig. 4. Time-resolved luminescence spectra of lateral emission of InGaN/GaN MQW structures grown (a) on GaN and (b) on sapphire (the spectra are arbitrary shifted along the vertical axis). 
Results on laterally amplified luminescence evidently show that the heteroepitaxially grown structure can be characterized by a significantly higher variation in the In content, which results in a smeared density of states. The stimulated and spontaneous emission bands are separated in time and energy scales. Spontaneous emission occurs within the tail of the density of states on the late stage of relaxation, while the stimulated emission occurs close to mobility edge, immediately after short-pulse excitation. Compositional disorder results in a decrease in the effective density of states, which leads to a higher lasing threshold and a blue-shift of stimulated emission. The resulting energy separation between the spontaneous and stimulated emission bands is about $20 \mathrm{meV}$ for the homoepitaxially grown sample and about $200 \mathrm{meV}$ for the heteroepitaxially grown one. The only difference between two samples is the density of threading dislocations, which is significantly lower for homoepitaxially grown structures. Thus threading dislocations can play a key role in producing compositional inhomogeneity in InGaN/GaN MQWs. Moreover, owing to threading dislocations and compositional disorder, the structure grown on sapphire can have larger diffractive losses, which is evidenced by the more than two orders of magnitude lower efficiency of laterally amplified emission observed at high excitation.

\section{Conclusions}

InGaN/GaN MQWs grown on GaN and sapphire substrates were characterized by luminescence spectroscopy under high excitation conditions, close to those of semiconductor laser operation regime. Transient and dynamic properties of luminescence indicate a significant reduction in compositional disorder in the homoepitaxially grown structures. This results in a significantly lower threshold of stimulated emission and thus in an enhanced intensity of the lateral luminescence for InGaN/GaN MQWs grown over bulk GaN substrate.

\section{Acknowledgments}

The research at Vilnius University was partially supported by the Lithuanian State Science and Education Foundation under COST529 program and European Commission supported SELITEC center Contract No. G5MA-CT-200204047. A.Ž. acknowledges the Lithuanian Ministry of Education and Science for his fellowship.

\section{References}

[1] S. Nakamura, S.F. Chichibu, Introduction to Nitride Semiconductor Blue Lasers and Light Emitting Diodes, Taylor \& Francis, London 2000.

[2] A. Žukauskas, M.S. Shur, R. Gaska, Introduction to Solid-State Lighting, Wiley, New York 2002. 
[3] H. Sato, T. Sugahara, Y. Naoi, S. Sakai, Jpn. J. Appl. Phys. 37, 2013 (1998).

[4] W.S. Wong, M. Kneissl, P. Mei, D.W. Treat, M. Teepe, N.M. Johnson, Appl. Phys. Lett. 78, 1198 (2001).

[5] D.G. Zhao, S.J. Xu, M.H. Xie, S.Y. Tong, H. Yang, Appl. Phys. Lett. 83, 677 (2003).

[6] T. Egawa, H. Ohmura, H. Ishikawa, T. Jimbo, Appl. Phys. Lett. 81, 292 (2002).

[7] A. Yasan, R. McClintock, K. Mayes, S.R. Darvish, H. Zhang, P. Kung, M. Razeghi, S.K. Lee, J.Y. Han, Appl. Phys. Lett. 81, 2151 (2002).

[8] E. Kuokstis, J.W. Yang, G. Simin, M.A. Khan, R. Gaska, M.S. Shur, Appl. Phys. Lett. 80, 977 (2002).

[9] K. Omae, Y. Kawakami, S. Fujita, Y. Narukawa, T. Mukai, Phys. Rev. B 68, 085303 (2003).

[10] S. Miasojedovas, S. Juršènas, G. Kurilčik, A. Žukauskas, Y.-C. Cheng, T.-Y. Tang, C.C. Yang, C.-T. Kuo, J.-S. Tsang, Phys. Status Solidi C 0, 2610 (2003). 